\title{
Targeting cholesterol-rich microdomains to circumvent tamoxifen-resistant breast cancer
}

\author{
Richa Tiwary ${ }^{1 \dagger}$, Weiping $\mathrm{Yu}^{1 \dagger}$, Linda A deGraffenried ${ }^{2}$, Bob G Sanders ${ }^{1}$ and Kimberly Kline ${ }^{2^{*}}$
}

\begin{abstract}
Introduction: Adjuvant treatment with tamoxifen substantially improves survival of women with estrogen-receptor positive (ER+) tumors. Tamoxifen resistance (TAMR) limits clinical benefit. RRR- $\alpha$-tocopherol ether-linked acetic acid analogue $(\alpha$-TEA) is a small bioactive lipid with potent anticancer activity. We evaluated the ability of $\alpha$-TEA in the presence of tamoxifen to circumvent TAMR in human breast cancer cell lines.
\end{abstract}

Methods: Two genotypically matched sets of TAM-sensitive (TAMS) and TAM-resistant (TAMR) human breast cancer cell lines were assessed for signal-transduction events with Western blotting, apoptosis induction with Annexin V-FITC/PI assays, and characterization of cholesterol-rich microdomains with fluorescence staining. Critical involvement of selected mediators was determined by using RNA interference and chemical inhibitors.

Results: Growth-factor receptors (total and phosphorylated forms of HER-1 and HER-2), their downstream prosurvival mediators pAkt, pmTOR, and pERK1/2, phosphorylated form of estrogen receptor- $\alpha$ (pER- $\alpha$ at Ser-167 and Ser-118, and cholesterol-rich lipid microdomains were highly amplified in TAMR cell lines and enhanced by treatment with TAM. $\alpha$-TEA disrupted cholesterol-rich microdomains, acted cooperatively with TAM to reduce prosurvival mediators, and induced DR5-mediated mitochondria-dependent apoptosis via an endoplasmic reticulum stress-triggered pro-death pJNK/CHOP/DR5 amplification loop. Furthermore, methyl- $\beta$-cyclodextrin $(M \beta C D)$, a chemical disruptor of cholesterol rich microdomains, acted cooperatively with TAM to reduce prosurvival mediators and to induce apoptosis.

Conclusions: Data for the first time document that targeting cholesterol-rich lipid microdomains is a potential strategy to circumvent TAMR, and the combination of $\alpha$-TEA + TAM can circumvent TAMR by suppression of prosurvival signaling via disruption of cholesterol-rich lipid microdomains and activation of apoptotic pathways via induction of endoplasmic reticulum stress.

\section{Introduction}

Of the estimated 207,090 new cases of breast cancer diagnosed among women in the United States in 2010, approximately $70 \%$ were ER+ [1]. Unfortunately, $40 \%$ to $50 \%$ of ER+ breast cancer patients either will not respond to endocrine therapy (that is, exhibit de novo resistance) or will have cancer recurrence because of acquired endocrine therapy resistance [2]. Clearly, more basic science information and different treatment regimens are needed to circumvent endocrine therapy resistance.

\footnotetext{
* Correspondence: k.kline@mail.utexas.edu

† Contributed equally

${ }^{2}$ Department of Nutritional Sciences/A2703, University of Texas at Austin, 1 University Station, Austin, TX 78712, USA

Full list of author information is available at the end of the article
}

TAM is a selective estrogen-receptor modulator with estrogenic actions in endometrial tissue, adipose tissue, and bone, and anti-estrogenic actions in breast tissue [3]. TAM, which binds to ER- $\alpha$ and antagonizes ER- $\alpha$ actions in breast tissue, has been the mainstay of endocrine therapy in both early and advanced ER+ breast cancer patients for almost three decades. However, TAM resistance remains the major barrier for its successful application in the clinic. De novo and acquired resistance may occur through altered cell-signaling mediators, leading to estrogen-independent activation of ERmediated gene expression and hormone independence [4]. Of the many events producing TAMR, aberrant overexpression of prosurvival signaling is implicated as an important contributor to both acquired and de novo TAMR [5,6]. TAMR cells have been shown to

\section{() Biomed Central}


overexpress receptor tyrosine kinases (RTKs), such as HER-1 and HER-2, and to crosstalk with membraneassociated ER (mER), leading to nuclear estrogen-receptor (nER) dependent and independent cell proliferation in which TAM acts as an agonist [6-8].

Cholesterol-enriched lipid-raft microdomains are characterized as lateral assemblies of glycosphingolipids and cholesterol that form liquid-ordered membrane phases with detergent-resistant structures. Cholesterol-enriched domains are highly expressed in tumor cells $[9,10]$ and provide the necessary platforms for growth factors, RTKs, and their downstream mediators, such as Akt and ERK (RTKs/Akt and RTKs/ERK complexes), to interact and crosstalk, leading to cell proliferation and survival $[10,11]$. Therefore, cholesterol-enriched lipidraft domains are described as "survival pools" for promoting prosurvival and pro-proliferation pathways, both of which are targets for cancer prevention and therapy.

$\alpha$-TEA, a unique small pleiotropic-acting lipid, has been shown to possess anticancer properties that are selective for cancer cells and not normal cells and that are nontoxic both in vitro and in vivo [12-24]. Mechanistic studies show that $\alpha$-TEA has two major effects that are necessary and sufficient for inducing apoptosis of cancer cells: (a) activation of proapoptotic pathways including Fas receptor (FasR)/Fas ligand (Fas L), endoplasmic reticulum stress-mediated JNK/CHOP/DR5 and p73/Noxa, leading to caspase- 8 and mitochondriadependent apoptosis, and (b) suppression of prosurvival/ antiapoptotic factors such as HER-1, HER-2, Akt, ERK, cellular FLICE-inhibitory protein (c-FLIP), and B-cell lymphoma 2 (Bcl-2), and survivin [13,18-24]. Additionally, $\alpha$-TEA has been shown to stimulate antitumor immune responses [25].

Data presented here show that $\alpha$-TEA circumvents TAMR in the presence of TAM via activation of endoplasmic reticulum stress-mediated DR5-dependent proapoptotic signaling and disruption of cholesterol-rich microdomains, leading to downregulation of prosurvival pathways.

\section{Materials and methods Chemicals}

$\alpha$-TEA (F.W. $=488.8$ ) was prepared in our laboratory as previously described [16]. Tamoxifen was purchased from Calbiochem (La Jolla, CA). Filipin, methyl- $\beta$-cyclodextrin $(\mathrm{M} \beta \mathrm{CD})$ and cholesterol were purchased from Sigma (St. Louis, MO). Dialkylindocarbocyanine (DilC16) was purchased from Molecular Probes (Eugene, OR).

\section{Cell culture and treatments}

TAM-sensitive MCF-7/parental (MCF-7/TAMS) and acquired tamoxifen-resistant MCF-7 (MCF-7/TAMR) cells were a gift from Dr. Linda A. deGraffenried
(University of Texas at Austin). MCF-7/TAMS cells were cultured as previously described [23]. MCF-7/TAMR cells were grown in phenol-red-free improved modified Eagle's medium (IMEM) with $10 \%$ charcoal stripped (steroid-depleted) serum supplemented with TAM $\left(10^{-7}\right.$ $M)$. [Note: TAM is required to maintain the TAMR phenotype of MCF-7/TAMR cells in culture]. Three days before treatment, cells were grown in phenol-red-free IMEM with $10 \%$ charcoal-stripped serum supplemented with $17-\beta$-estradiol $\left(10^{-9} M\right)$ for MCF-7/TAMS and TAM $\left(10^{-7} M\right)$ for MCF-7/TAMR. Clone 18 MCF-7 cells overexpressing HER-2 (MCF-7/HER-2) that exhibit a TAMR phenotype [8] and their vector control (MCF-7/ Neo) cells (gifts from Dr. Mien-Chie Hung, MD Anderson, Houston, TX) were cultured under the same condition as MCF-7/TAMS. During treatments, serum was reduced to $2 \%$ without TAM. Cells were treated with various concentrations of TAM, $\alpha$-TEA, or vehicle (VEH) control. Neither TAM nor $\alpha$-TEA is water soluble, so they are solubilized in ethanol before addition to media and an ethanol control (called vehicle), consisting of the highest concentration of ethanol used for solubilization in a given experiment, was included as a control.

\section{Western blot analyses}

Western blot analyses were conducted as described previously [22]. Antibodies to the following proteins were used: poly (ADP-ribose) polymerase (PARP), c-FLIP, CHOP, glucose-regulated protein (GRP-78), pERK, total ERK (tERK), and pJNK (Santa Cruz Biotechnology, Santa Cruz, CA), Bcl-2, caspase-8, caspase-9, DR5, phosphoHER-1 (pHER-1), total HER-1 (tHER-1), phospho-HER-2 (pHER-2), total HER-2 (tHER-2), pER- $\alpha$ (Ser-118), pER$\alpha$ (Ser-167), total ER- $\alpha$ (tER- $\alpha$ ), pAkt (Ser-473), total Akt (tAkt), and glyceraldehyde-3-phosphate dehydrogenase (GAPDH) (Cell Signaling Technology, Beverly, MA).

\section{RNA interference}

A scrambled RNA duplex that does not target any known gene was used as the nonspecific negative control for RNA interference (referred to as control siRNA). Transfection of MCF-7/TAMR cells with siRNAs to DR5, CHOP, JNK, Akt-1, c-FLIP, or control (Ambion, Austin, TX) was performed as previously described [22].

\section{Quantification of apoptosis}

Apoptosis was quantified with the Annexin V-FITC/PI assay (Invitrogen, Carlsbad, CA) by following manufacturer's instructions. Data were analyzed by using CellQuest software (BD Biosciences, San Jose, CA).

\section{Staining with fluorescent-labeled DilC-16 and Filipin}

Fluorescein-labeled lipid analogue DilC-16, a lipid microdomain marker [26], and fluorescein-labeled 
Filipin, a cholesterol marker [27], were used to determine the presence of cholesterol-enriched lipid microdomains. For DilC-16 staining, cells were trypsinized and washed with phosphate-buffered saline (PBS) and stained with DilC-16 $(5 \mu M)$ for 15 minutes at room temperature. For filipin staining, cells were trypsinized, washed with PBS, fixed with 3\% paraformaldehyde for 30 minutes at room temperature, rinsed with PBS, and incubated with $1.5 \mathrm{mg} / \mathrm{ml}$ glycine in PBS to quench the paraformaldehyde. Cells were then stained with fluorescein-labeled filipin $(50 \mu \mathrm{g} / \mathrm{ml})$ for 1 hour at room temperature and washed with PBS. Cells were viewed by using a fluorescence microscope with TRITC filter setting for DiLC-16 staining and UV filter setting for filipin staining, respectively.

\section{Statistical analysis}

One-way analysis of variance (ANOVA) followed by Tukey test was used for comparison of more than two treatments to determine statistical differences. Differences were considered statistically significant at $P<0.05$.

\section{Results}

TAMR cells in comparison with TAMS cells constitutively express higher levels of prosurvival mediators and cholesterol-enriched lipid microdomains

Total and phosphorylated protein profiles of prosurvival mediators in both de novo and acquired TAMR cell lines (MCF-7/HER-2 and MCF-7/TAMR, respectively), cultured with and without TAM, in comparison with their parental TAMS cells, were determined by Western blot analyses. Growth-factor receptors HER-1 and HER2 (phosphorylated form as well as total protein) and their downstream mediators pAkt and pERK1/2, as well as pER- $\alpha$ (Ser-167 and Ser-118) are expressed at markedly higher levels in TAMR cells in comparison with their parental TAMS cells (Figure 1a). pHER-1 and pHER-2 were below levels of detection in the TAMS cell lines either with or without TAM treatment (Figure 1a). As expected, TAM treatment reduced levels of activated Akt (pAkt) and activated ER- $\alpha$ (pER- $\alpha$ Ser-167 and Ser-118) in both TAMS cell lines, but had the opposite effect on the TAMR cells, measurably enhancing activated pAkt and pER- $\alpha$ (Ser-167 and Ser-118) in TAMR cells (Figure 1a). These data document that prosurvival signaling is constitutively highly expressed in TAMR cells in comparison with TAMS cells, and that TAM treatment differentially affects prosurvival signaling between TAMS and TAMR cells; TAM downregulates prosurvival mediators in TAMS cells and increases them in TAMR cells. Furthermore, both TAMR cell lines express higher levels of the fluorescent lipid analogue DilC-16, a marker of lipid microdomains, and fluorescein-labeled filipin, a cholesterol marker, as viewed by

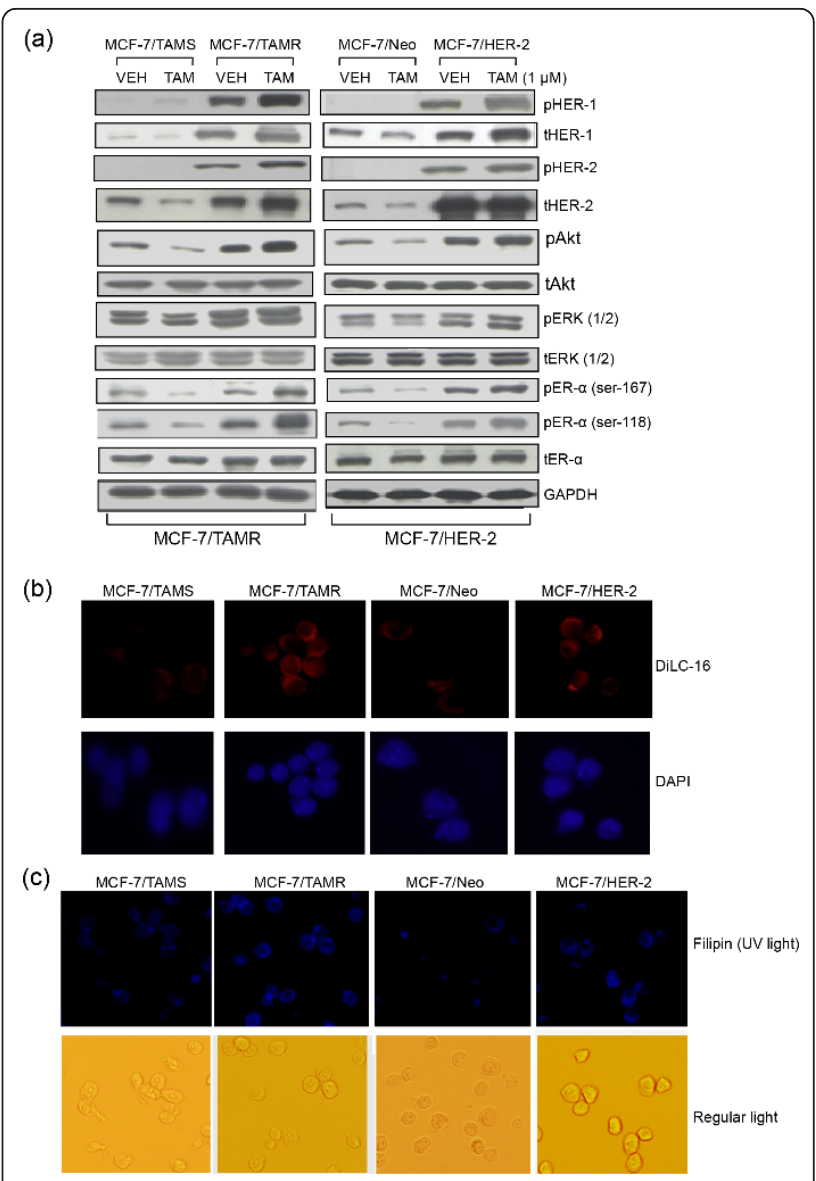

Figure 1 TAMR cells in comparison to TAMS cells constitutively express higher levels of prosurvival mediators and cholesterolrich lipid microdomains. (a) TAMR cell lines MCF-7/TAMR and MCF-7/HER-2, as well as TAMS parental MCF-7/TAMS and MCF-7/ Neo cells, were treated with TAM at $1 \mu M$ or VEH (ethanol) in steroid-depleted media containing $17 \beta$-estradiol $\left(10^{-9} \mathrm{M}\right)$ for 2 days. Molecular profile of prosurvival mediators was determined with Western blot analyses. (pHER-1/tHER-1 and pHER-2/tHER-2 were not detected in the MCF-7/TAMS and MCF-7/Neo cells). (b) The expression of lipid microdomains was determined by staining the cells with fluorescein-tagged DiLC16 lipid-raft marker and viewed with a fluorescence microscope. (c) The expression of cholesterolrich lipid microdomains was determined by staining cells with fluorescein-labeled filipin, a cholesterol marker, and viewed by using a fluorescence microscope. (a-c) Data are representative of a minimum of three independent experiments. DilC-16,

dialkylindocarbocyanine; HER, human epidermal growth factor; MCF7/HER-2, Clone 18 MCF-7 cells overexpressing HER-2; MCF-7/TAMR, acquired tamoxifen-resistant MCF-7; MCF-7/TAMS, TAM-sensitive MCF-7/parental; pHER-1, phosphorylated-HER-1; pHER-2,

phosphorylated-HER-2; TAM, tamoxifen; TAMR, tamoxifen resistant; TAMS, tamoxifen sensitive; VEH, vehicle control.

using a fluorescence microscope in comparison with TAMS cells (Figure $1 \mathrm{~b}$ and $1 \mathrm{c}$ ), suggesting that TAMR cells constitutively express higher levels of cholesterolenriched lipid rafts that are supportive of prosurvival signaling. 
$M B C D$ plus TAM treatment circumvents TAMR via induction of apoptosis and suppression of proliferation/ survival signaling

To determine whether cholesterol-rich lipid microdomains play a critical role in elevated expression of prosurvival mediators in TAMR cells, TAMR cells were cultured with the cholesterol-depleting agent $\mathrm{M} \beta \mathrm{CD}$ followed by analyses of proliferation/survival mediators. $\mathrm{M} \beta C D$ at 2.5 and $5 \mu M$ suppressed levels of total and pHER-1 and pHER-2, and decreased levels of pAkt and pER- $\alpha$ (Ser-118 and Ser-167) in MCF-7/TAMR cells (Figure $2 \mathrm{a}$ ). $\mathrm{M} \beta \mathrm{CD}$ at 1.25 or $2.5 \mu M$ in the presence of 0.5 or $1 \mu M$ TAM acted cooperatively to induce apoptosis significantly in both MCF-7/TAMR and MCF-7/ HER-2 cells in comparison with single treatments, as determined with Annexin V-FITC/PI analyses (Figure $2 \mathrm{~b})$ and cleavage of PARP, an indicator of apoptosis (Figure 2c). Furthermore, although $1 \mu M$ TAM treatment produced a trend toward increased levels of proliferation/survival mediators, $\mathrm{M} \beta \mathrm{CD}$ alone produced a trend of decreased expression of proliferation/survival mediators, and the combination of $\mathrm{M} \beta \mathrm{CD}+\mathrm{TAM}$ acted cooperatively to produce the most marked reduction in proliferation/survival mediators (Figure 2c), indicating that $M \beta C D$ restores TAM sensitivity. Taken together, these data demonstrate that $\mathrm{M} \beta \mathrm{CD}$ disruption of cholesterol-rich lipid microdomains circumvents TAMR when combined with TAM via suppression of prosurvival signaling and induction of apoptosis, providing additional support that cholesterol-enriched lipid microdomains participate in TAM resistance via enhancing proliferation/prosurvival signaling in TAMR cells.

$\alpha$-TEA cooperates with TAM to induce apoptosis in TAMR cell lines

$\alpha$-TEA induces apoptosis in a dose-dependent manner in both TAMR and TAMS cells (Figure 3a). $\alpha$-TEA treatment of MCF-7/TAMR and MCF-7/HER-2 at 10, 20 , and $40 \mu M$ significantly induced apoptosis in comparison with VEH control. As expected, TAM induced apoptosis only in TAM-sensitive MCF-7/parental cells, and not in either of the TAM-resistant cell lines (neither TAMR MCF-7/TAMR nor MCF-7/HER-2 cells (data not shown). To determine whether TAM can act cooperatively with $\alpha$-TEA to trigger TAM-resistant cells to undergo apoptosis, we examined the combination effect of three nonapoptotic doses of tamoxifen $(0.5,1$, and $1.5 \mu M)$ with three sub-half-maximal effective concentration $\left(\mathrm{EC}_{50}\right)$ apoptotic doses of $\alpha$-TEA $(10,20$, and 30 $\mu M)$ on induction of apoptosis. The combination of $\alpha$ TEA at 10,20 , and $30 \mu M$ plus TAM at $0.5,1$, and 1.5 $\mu M$, respectively, significantly increased the levels of apoptosis (Figure $3 \mathrm{~b}$ ) and cleaved PARP (c-PARP)
(Figure 3c) in MCF-7/TAMR and MCF-7/HER-2 cells in comparison with $\mathrm{VEH}$ control and single treatments.

We determined the proapoptotic effect obtained with TAM and $\alpha$-TEA combination by using the CalcuSyn (Biosoft, Manchester, UK) software package, which is designed to calculate combination indexes (CIs) by using the Chou-Talalay method for drug-combination efficacy based on the median-effects equation [28]. CI values of $0.59 \pm 0.00$ and $0.81 \pm 0.13$ indicated synergistic actions of $\alpha$-TEA + TAM (20:1 ratio) on induction of apoptosis in MCF-7/TAMR and MCF-7/HER-2 cell lines, respectively (Table 1 ). The cooperative proapoptotic actions of the combination of $\alpha$-TEA plus TAM were further confirmed by measurement of increased levels of cleaved caspases- 8 and -9 (c-caspase- 8 and -9) (Figure 3c), suggesting that the combination of $\alpha$-TEA + TAM induces caspase- 8 and -9 mediated apoptosis in both TAMR cell lines.

\section{Combination of $\alpha$-TEA + TAM acted cooperatively to induce endoplasmic reticulum stress-mediated apoptosis} Because $\alpha$-TEA has been shown to induce endoplasmic reticulum stress [18], we wanted to explore the possibility that $\alpha$-TEA + TAM were inducing endoplasmic reticulum stress-mediated apoptosis. Combination of $20 \mu \mathrm{M}$ $\alpha$-TEA $+1 \mu M$ TAM induced increased levels of endoplasmic reticulum stress-associated proapoptotic factors, pJNK (2/1), CHOP, and DR5 long and short (L/S) and endoplasmic reticulum stress marker GRP78 in both TAMR cell lines (Figure 4a). siRNAs to CHOP, DR5, and JNK blocked the ability of the combination treatment to induce apoptosis, as determined by the absence of cleaved PARP and blockage of combination treatment-induced increases in pJNK (2/1), CHOP, and DR5 $(\mathrm{L} / \mathrm{S})$ in the MCF-7/TAMR cell line (Figure $4 \mathrm{~b})$, indicating that the combination of $\alpha$-TEA + TAM enhances $\alpha$ TEA-induced endoplasmic reticulum stress-mediated apoptosis, which involves JNK/CHOP/DR5.

\section{Combination of $\alpha$-TEA + TAM circumvents TAMR via cooperatively suppressing prosurvival/antiapoptotic factors}

As shown in Figure 1a, TAM induces the expression of prosurvival mediators HER-1 and HER-2 (total and phosphorylated forms), as well as pAkt, pERK2/1, and pER- $\alpha$ (Ser 167 and 118) in TAMR cells. Importantly, $\alpha$-TEA alone and, even more markedly, when in combination with TAM, reduced the expression of these proliferation/survival mediators (Figure 5a), indicating that $\alpha$-TEA restores TAM sensitivity in TAMR cells by downregulating survival factors. Moreover, TAM alone induced increased levels of antiapoptotic factors c-FLIP and Bcl-2 (Figure 5b), suggesting that these 


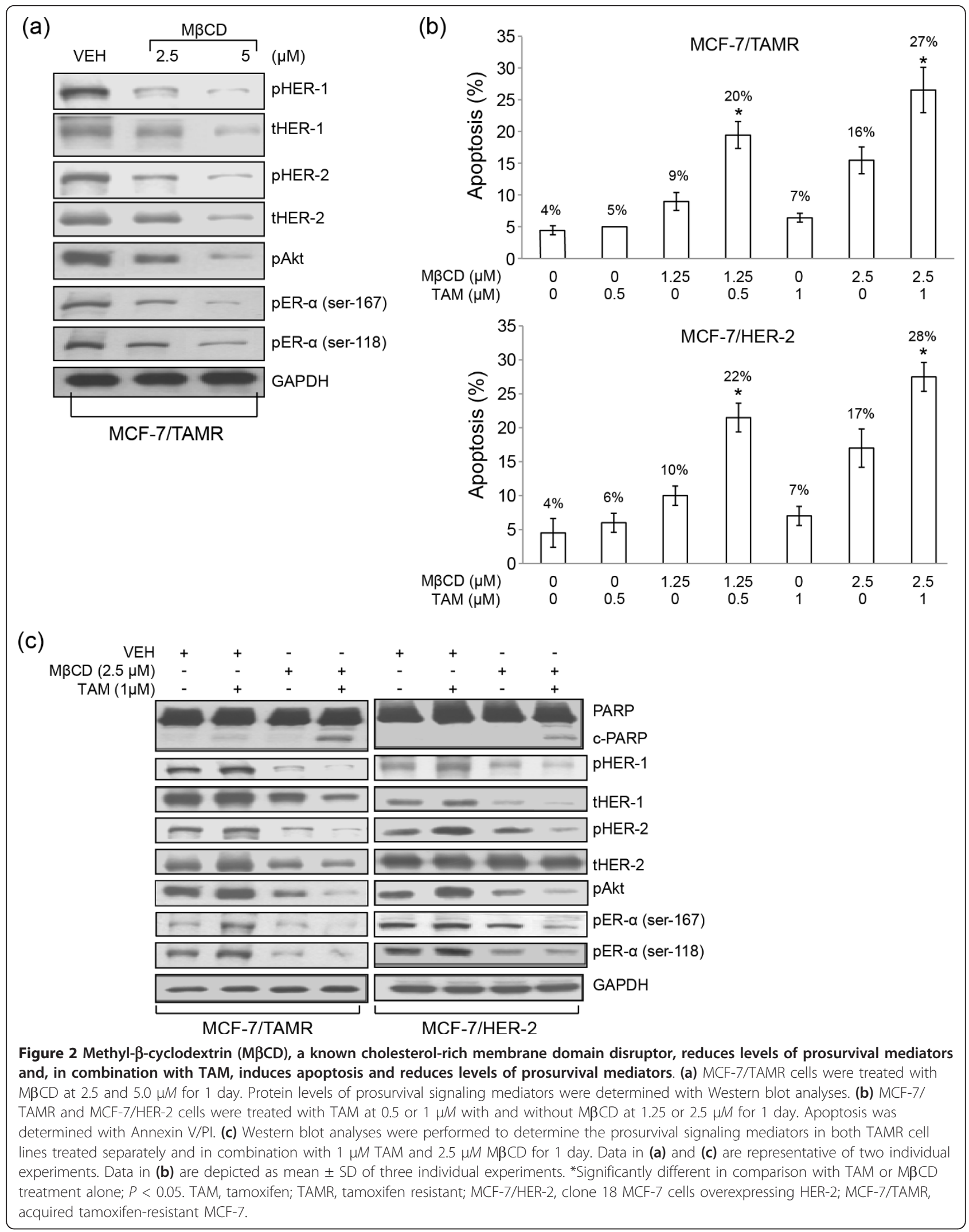


Table 1 Combination index of apoptosisa

\begin{tabular}{lllllll}
\hline & $\alpha-$ TEA:TAM & $\mathrm{Cl}^{\mathbf{c}}$ & & & & \\
\hline & Ratio $^{\mathbf{b}}$ & $\mathrm{ED}_{\mathbf{5 0}}$ & $\mathrm{ED}_{\mathbf{7 5}}$ & $\mathrm{ED}_{\mathbf{9 0}}$ & Mean $+\mathrm{SD}^{\mathbf{d}}$ & \\
MCF-7/TAMR & $20: 1$ & 0.59 & 0.59 & 0.59 & $0.59 \pm 0.00$ & Synergism $^{\text {a }}$ \\
MCF-7/HER-2 & $20: 1$ & 0.68 & 0.80 & 0.94 & $0.81 \pm 0.13$ & Synergism $^{\text {a }}$ \\
\hline
\end{tabular}

${ }^{a}$ MCF-7/TAMR and MCF-7/HER-2 human breast cancer cells were treated with different concentrations of $\alpha$-TEA and TAM alone and in combinations for 24 hours. Apoptosis was determined as described in Material and Methods. ${ }^{\mathrm{b}}$ The ratio for concentrations used in combination treatments were determined from data in Figure $3 \mathrm{~b}$ and $\mathrm{c}$. 'For each combination treatment, a combination index (Cl) was calculated by using methods as described previously and by using the commercially available software (CalcuSyn; Biosoft, Manchester, United Kingdom). ${ }^{\mathrm{d}}$ The mean \pm standard deviation (SD) is calculated from the Cl values of effective dosages (EDs) that produced $50 \%, 75 \%$, and $90 \%$ apoptosis. ${ }^{e} \mathrm{Cl}$ values $<1.0$ indicate synergism; $\mathrm{Cl}$ values of 1.0 indicate additive effect; and $\mathrm{Cl}$ values $>1.0$ indicate antagonism. $\alpha$-TEA, RRR- $\alpha$-tocopherol ether-linked acetic acid analogue; MCF-7/HER-2, clone 18 MCF-7 cells overexpressing HER-2; MCF-7/TAMR, acquired tamoxifen-resistant MCF-7; mER, membrane estrogen receptor; TAM, tamoxifen.

antiapoptotic factors may be mediated by prosurvival signaling. Again, the combination of $\alpha$-TEA + TAM acted cooperatively to suppress these antiapoptotic factors (Figure $5 b$ ).

In an effort to understand how TAM cooperates with $\alpha$-TEA to induce endoplasmic reticulum stress and endoplasmic reticulum stress-mediated JNK/CHOP/ DR5, we knocked down Akt-1 and c-FLIP by using siRNA to examine the impact on $\alpha$-TEA-mediated upregulation of JNK1/2, CHOP, DR5, and GRP-78 protein levels. siRNAs to Akt-1 and c-FLIP enhanced $\alpha$-TEAinduced apoptosis, as detected by PARP cleavage (Figure $5 c)$, as well as enhanced the $\alpha$-TEA ability to increase protein levels of JNK1/2, CHOP, DR5(L/S), and GRP-78 (Figure 5c). siRNA to Akt-1 reduced pAkt levels and reduced c-FLIP expression, and the combination of $\alpha$ TEA + siRNA to Akt-1 acted cooperatively to suppress pAkt further and to reduce c-FLIP expression (Figure 5c). siRNA to c-FLIP reduced c-FLIP protein levels, but not pAkt, and acted cooperatively with $\alpha$-TEA to reduce further the c-FLIP expression as well as to reduce pAkt levels (Figure 5c). These data suggest that c-FLIP is regulated, at least in part, by Akt-1, and downregulation of Akt/c-FLIP contributes to the $\alpha$-TEA ability to upregulate pJNK, CHOP, DR5, and GRP78. Taken together, data presented in Figure 5 demonstrate that the combination of $\alpha$-TEA + TAM acts cooperatively to suppress markedly both prosurvival and antiapoptotic signaling mediators.

\section{Reductions in cholesterol-rich lipid-raft domains are involved in $\alpha$-TEA + TAM circumvention of TAMR}

Cholesterol-rich lipid microdomains support cell proliferation and cell survival. As detected by staining cells with the cholesterol marker filipin, treatment with $\alpha$ TEA in comparison with VEH control produced reductions in cholesterol-rich microdomains (Figure 6a). Pretreatment of MCF-7/TAMR cells with $10 \mu M$ exogenous cholesterol, an established method for enhancing cholesterol-rich microdomains [29] for 2 hours blocked the ability of both $\alpha$-TEA alone and the combination of $\alpha$-TEA + TAM to induce apoptosis, as detected by PARP cleavage (Figure 6b); as well as to decrease protein levels of prosurvival signaling mediators (Figure $6 \mathrm{~b}$ ). These data suggest that cholesterol-rich lipid microdomains are important for $\alpha$-TEA + TAM circumvention of TAMR.

\section{Discussion}

Acquired and de novo tamoxifen resistance are major barriers for successful application of tamoxifen in the clinic. Data reported here document that TAMR cells constitutively express highly elevated growth-factor signaling mediators that can be depleted by reducing cholesterol-rich microdomains and that $\alpha$-TEA, a small bioactive lipid, in combination with TAM, restores TAM sensitivity to TAMR cells via suppression of TAMR proliferation/survival mediators and induction of cell death by apoptosis. Novel findings from these studies are as follows: (a) TAMR cells express higher levels of cholesterol-rich lipid microdomains than do TAMS cells; (b) disrupting cholesterol-rich lipid microdomains with the cholesterol-depleting agent $\mathrm{M} \beta \mathrm{CD}$ suppressed TAMR prosurvival signaling and induced apoptosis when combined with TAM; (c) treating TAMR cells with the unique anticancer agent $\alpha$-TEA alone reduced cholesterol-rich lipid microdomains, reduced levels of constitutively expressed pro-proliferation/prosurvival signaling mediators, and led to apoptosis via endoplasmic reticulum stress-mediated JNK/CHOP/DR5 signaling; (d) the combination of $\alpha$-TEA + TAM had the best impact on circumventing TAMR via decreased expression of prosurvival/antiapoptotic mediators and induction of endoplasmic reticulum stress-mediated JNK/ CHOP/DR5 proapoptotic mediators; and (e) suppression of constitutively expressed pAkt or c-FLIP in cells by siRNA enhanced $\alpha$-TEA-induced apoptosis; as well as endoplasmic reticulum stress-mediated JNK/CHOP/DR5 signaling, indicating an important role for crosstalk between prosurvival Akt/antiapoptotic c-FLIP and the pro-death endoplasmic reticulum stress pathway.

Taken together, our data for the first time demonstrate that reducing cholesterol-rich lipid microdomains is a promising strategy for circumventing TAMR in 

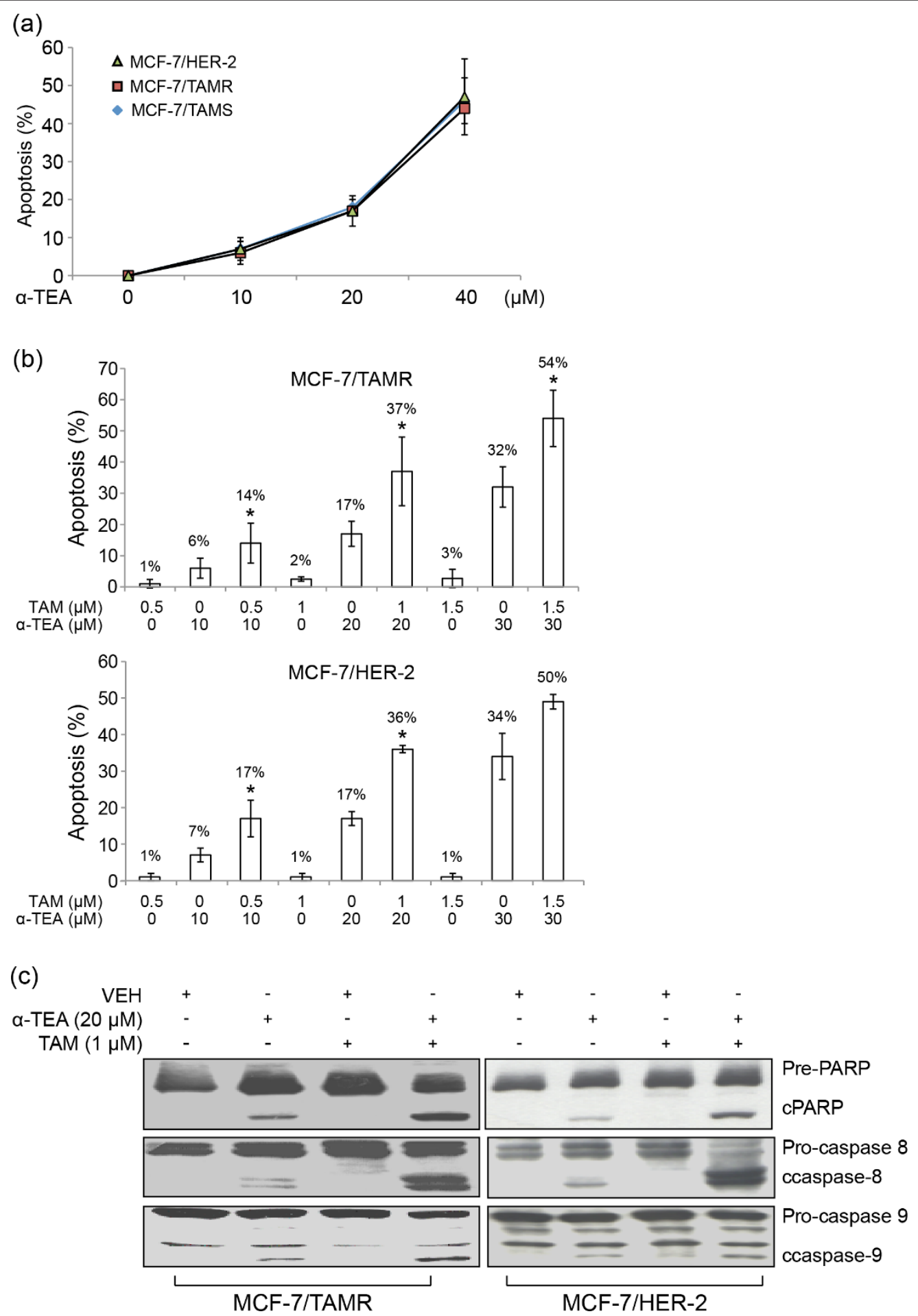

Figure $3 \alpha$-TEA alone and in combination with TAM induces apoptosis in TAMR cell lines.(a) TAM-sensitive MCF-7/parental cells and TAMR MCF-7/TAMR and MCF-7/HER-2 cells were treated with 10, 20, and $40 \mu M \alpha$-TEA for 1 day. Apoptosis was determined with Annexin V/PI. (b) MCF-7/TAMR and MCF-7/HER-2 cells were treated with 10 or 20 or $30 \mu M \alpha$-TEA, 0.5 or 1 or $1.5 \mu M$ TAM, and in combination for 1 day. Apoptosis was determined with annexin V/PI/FACS assay. (c) MCF-7/TAMR and MCF-7/HER-2 cells were treated separately and in combination with $20 \mu M \alpha$-TEA and $1 \mu M$ TAM for 1 day. Western blot analyses were performed to determine cleaved (c-) forms of caspases-8, 9, and PARP. Data in (a) and (b) are depicted as mean \pm SD of three individual experiments. *Significantly different in comparison with TAM and $\alpha$-TEA alone; $P<0.05$. Data in (c) are representative of three individual experiments. $\alpha$-TEA, RRR- $\alpha$-tocopherol ether-linked acetic acid analogue; HER-2, epidermal growth-factor receptor-2 ErbB-2; TAM, tamoxifen; TAMR, tamoxifen resistant; MCF-7/HER-2, clone 18 MCF-7 cells overexpressing HER-2; MCF-7/TAMR, acquired tamoxifen-resistant MCF-7. 


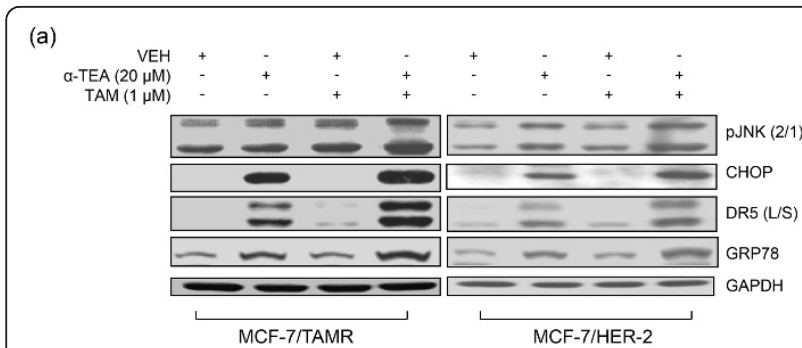

(b)

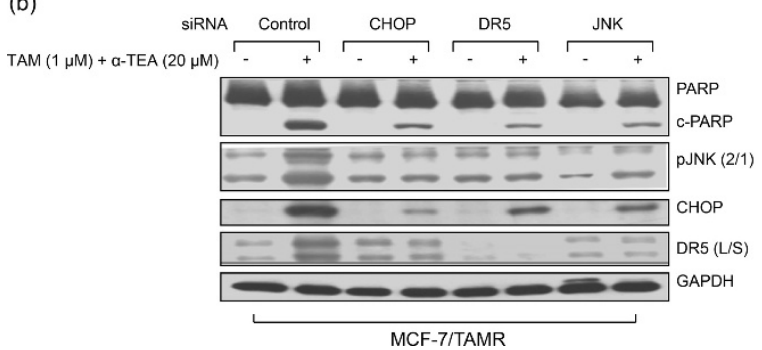

Figure $4 \alpha$-TEA alone and in combination with TAM induces biomarkers of endoplasmic reticulum stress, and siRNA knockdowns show necessary roles for CHOP, DR5, and JNK. (a) Western immunoblot analyses, using aliquots of cell lysates from cells treated with $20 \mu M \alpha$-TEA and $1 \mu M$ TAM in Figure 3b, were performed to assess pJNK2/1, CHOP, and DR5 (L/S) protein levels, as well as endoplasmic reticulum stress marker GRP78 protein expression with GAPDH as loading control. (b) MCF-7/TAMR cells transfected with siRNAs to CHOP, DR5, and JNK as well as control siRNA (labeled Control) were treated with TAM $(1 \mu M)+\alpha$-TEA (20 $\mu \mathrm{M})$ for 1 day. Western immunoblot analyses were performed to determine degree of apoptosis, as measured by cleaved PARP (CPARP), pJNK2/1, CHOP, and DR5 (L/S) protein levels, with GAPDH serving as the loading control. Data from (a) and (b) are representative of three individual experiments. CHOP, Ccaatenhancer-binding protein (C/EBP) homologous protein; DR5 (L/S), death receptor 5 long/short; GAPDH, glyceraldehyde-3-phosphate dehydrogenase; GRP78, glucose-regulated protein-78; JNK, c-Jun Nterminal kinase; MCF-7/TAMR, acquired tamoxifen-resistant MCF-7; PARP, poly (ADP-ribose) polymerase; siRNA, small interfering RNA; TAM, tamoxifen; TAMR, tamoxifen resistant; $\alpha$-TEA, RRR- $\alpha$-tocopherol ether-linked acetic acid analogue.

human breast cancer cells and that the combination of $\alpha$-TEA + TAM is a potentially beneficial regimen for preventing and circumventing TAMR in human breast cancer.

It is well established that TAMR is caused by overexpression of receptor tyrosine kinases (RTKs) proteins such as HER-1 and HER-2, which enhance RTK crosstalk with membrane-associated estrogen receptor, resulting in ER-independent and -dependent cell proliferation and survival via their downstream mediators Akt and ERK. Akt and ERK regulate multiple prosurvival factors; as well as activate nuclear ER- $\alpha$ via phosphorylation of ER- $\alpha$ at Ser-118 by ERK and Ser-167 by Akt $[7,30]$. Furthermore, it is well established that TAM acts as an agonist in TAMR cells stimulating prosurvival signaling

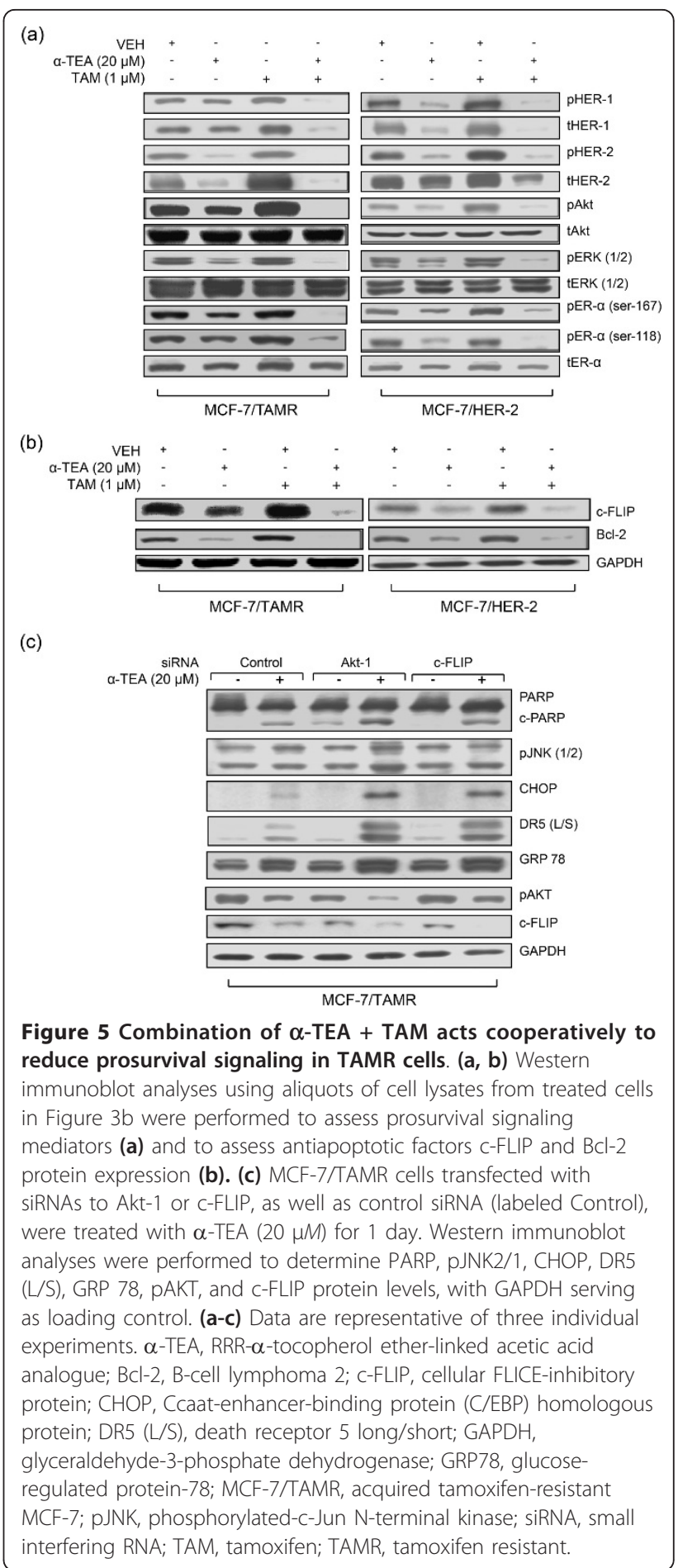

$[7,30]$. Thus, the combination of constitutively highly expressed prosurvival mediators and TAM, acting as an agonist rather than antagonist, are key molecular features of both de novo and acquired ER+ TAMR cells. Targeting various components in this highly amplified prosurvival signaling context, such as HER-1, HER-2, Akt, or mTOR, by using chemical inhibitors or 


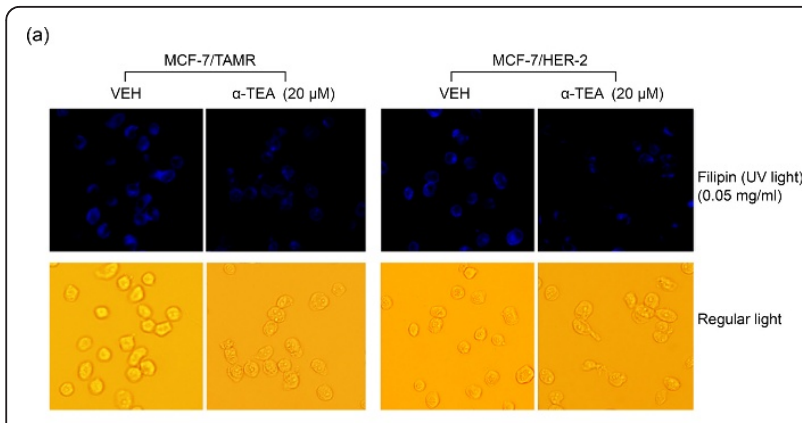

(b)

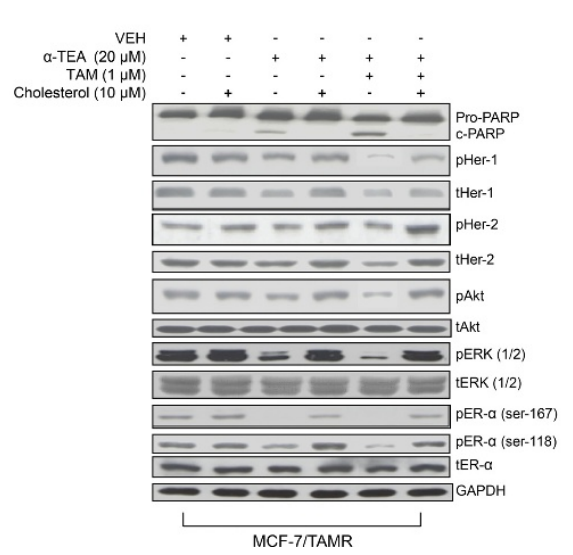

Figure 6 Cholesterol-rich lipid microdomains are involved in $\alpha$ TEA + TAM circumvention of TAMR. (a) MCF-7/TAMR and MCF-7/ HER-2 cells were treated with $\alpha$-TEA at $20 \mu M$ for 15 hours. Identification of cholesterol-rich microdomains was determined by staining the cells with fluorescein-tagged cholesterol marker Filipin and images viewed by using a fluorescence microscope. (b) MCF-7/ TAMR cells were pretreated with $10 \mu M$ exogenous cholesterol for 2 hours, followed by treatment with $20 \mu M \alpha$-TEA or $20 \mu M \alpha$-TEA plus 1 HM TAM for I day. Western blot analyses were performed to determine treatment effects on prosurvival signaling mediators and PARP cleavage. $(\mathbf{a}, \mathbf{b})$ Data are representative of three independent experiments. $\alpha$-TEA, RRR- $\alpha$-tocopherol ether-linked acetic acid analogue; MCF-7/HER-2, clone 18 MCF-7 cells overexpressing HER-2; MCF-7/TAMR, acquired tamoxifen-resistant MCF-7; PARP, poly (ADPribose) polymerase; TAM, tamoxifen; TAMR, tamoxifen resistant.

neutralizing antibodies has been reported to circumvent TAMR [31-34]. However, targeting single components, such as HER-1 or HER-2, eventually causes resistance $[35,36]$. Therefore, alternative approaches are required to circumvent TAMR.

Cholesterol-enriched lipid microdomains have been shown to provide platforms for crosstalk among growth factors, their receptors, and downstream mediators, leading to activation of prosurvival signaling $[10,11,37]$. It has also been reported that cholesterol-enriched lipid microdomains participate in crosstalk between $\mathrm{mER}$ and growth-factor receptors [38]. Furthermore, cholesterolrich membrane rafts have been hypothesized to provide privileged sites for nongenomic hormone signaling in prostate cancer cells, which could stimulate cell proliferation [39]. Therefore, disrupting cholesterol-rich lipid microdomains holds promise as a strategy for circumventing TAMR via downregulation of prosurvival signaling. However, little information exists on the role of cholesterol-rich lipid microdomains in TAMR. In this study, we examined whether alterations in the cholesterol content of lipid rafts in TAMR cell membranes affected cell-survival mediators. Here, for the first time, we report that TAMR cells expressed high levels of cholesterol-rich lipid microdomains, and that $\mathrm{M} \beta \mathrm{CD}$, a cholesterol-depleting agent that is used in research to disrupt lipid rafts, suppresses TAMR prosurvival signaling and circumvents TAMR when combined with TAM via restoration of TAM sensitivity and induction of apoptosis. These results implicate the necessity of cholesterol-enriched domains in survival of TAMR cells and suggest that agents that can disrupt cholesterol-enriched domains have potential as a promising strategy to circumvent TAMR when combined with TAM in ER+ breast cancer. Based on data presented here, $\alpha$-TEA is one such agent.

$\alpha$-TEA exerts its anticancer actions via activation of proapoptotic pathways and suppression of prosurvival pathways. However, molecular details of how $\alpha$-TEA affects these prosurvival/antiapoptotic factors are not fully understood. Previously, we reported that $\alpha$-TEA downregulates phosphatidylinositol-3-kinase (PI3K)/Akt/ ERK pathways via JNK-mediated downregulation of insulin-receptor substrate (IRS-1) [20]. Data presented here suggest that disruption of cholesterol-rich lipid microdomains may be another mechanism of $\alpha$-TEA action. The data to support this notion come from data presented here showing the following: (a) $\alpha$-TEA disrupts cholesterol-rich lipid microdomains; (b) addition of exogenous cholesterol to enrich lipid microdomains further, blocks the ability of $\alpha$-TEA to suppress prosurvival mediators; and (c) $\alpha$-TEA acts in a cooperative manner with $\mathrm{M} \beta C D$, a cholesterol disruptor, more markedly to suppress TAMR prosurvival signaling. How $\alpha$-TEA disrupts cholesterol-rich lipid microdomains is not known. Because $\alpha$-TEA has been reported to enhance ceramide accumulation in cellular membranes [40] and ceramide-enriched lipid microdomains have been reported to disrupt cholesterol lipid microdomains [41], one possibility is that $\alpha$-TEA disrupts cholesterolrich plasma membrane domains by increasing ceramiderich lipid microdomains.

Mechanistically, both $\mathrm{M} \beta C D$ and $\alpha$-TEA cooperated with TAM to suppress TAMR prosurvival signaling, leading to TAM-induced apoptosis. Both agents reduced cholesterol-rich lipid microdomains, which was demonstrated to be critical to both $M \beta C D$ and $\alpha$-TEA circumvention of TAMR. However, although both $M \beta C D$ and 


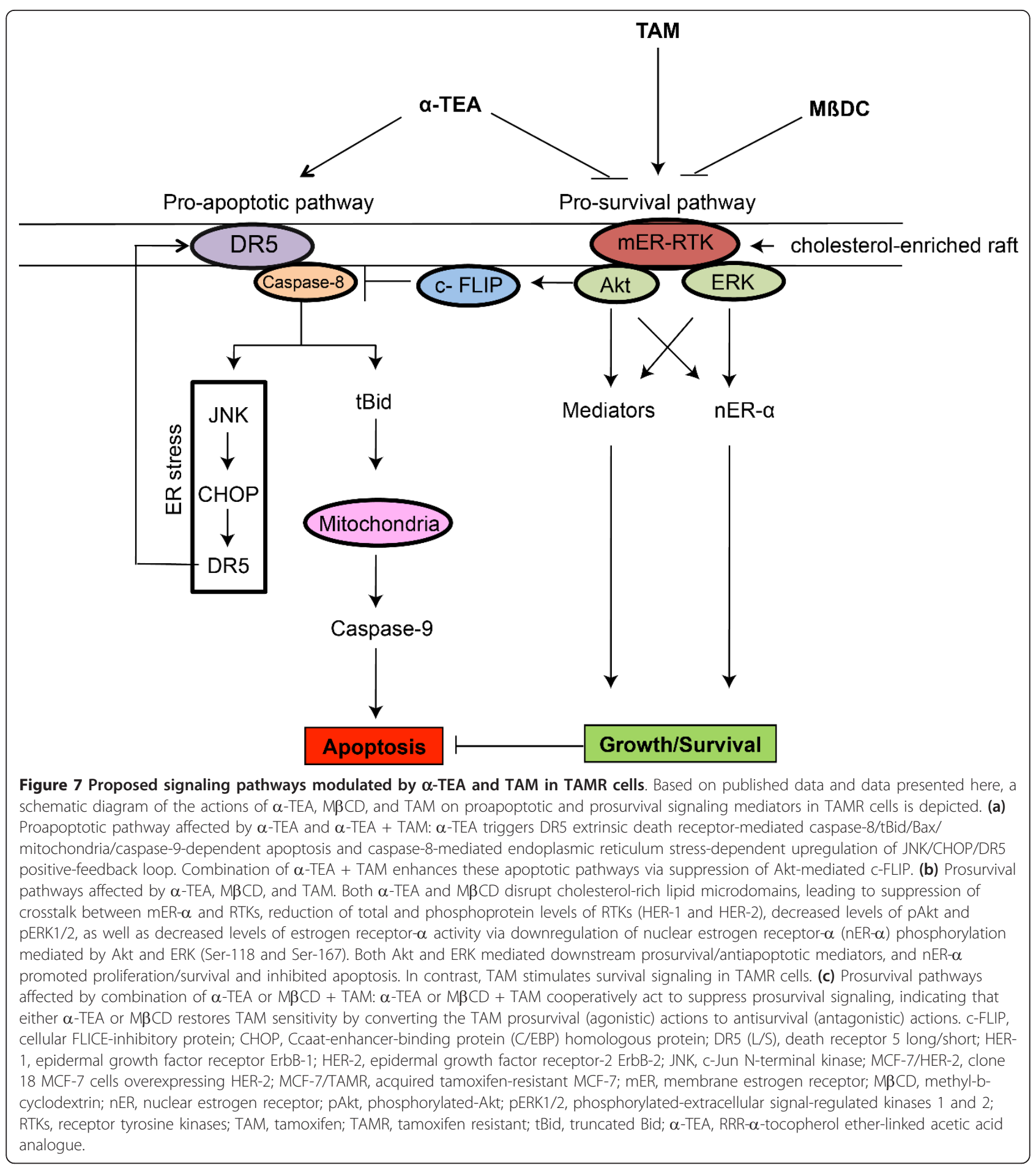

$\alpha$-TEA cooperated with TAM to induce apoptosis in TAMR cells the mechanisms are not the same. $\alpha$-TEA + TAM induces endoplasmic reticulum stress-mediated JNK/CHOP/DR5 proapoptotic events, whereas M $\beta C D+$ TAM did not induce endoplasmic reticulum stress (data not shown). In summary, $\alpha$-TEA + TAM induces apoptosis not only via suppression of prosurvival pathways, but also via activation of endoplasmic reticulum stressmediated pro-apoptotic events, demonstrating that the $\alpha$-TEA + TAM combination is a unique regimen for circumvention of TAMR.

How TAM cooperatively acts with $\alpha$-TEA to induce endoplasmic reticulum stress-mediated JNK/CHOP/DR5 is not entirely clear. One possibility is that crosstalk 
occurs between $\alpha$-TEA downregulation of prosurvival signaling and induction of endoplasmic reticulum stress. Published data show that downregulation of c-FLIP can enhance the $\alpha$-TEA-induced endoplasmic reticulum stress proapoptotic pathway via activation of caspase-8, because caspase- 8 is involved in $\alpha$-TEA-induced endoplasmic reticulum stress [18]. Data presented here show that siRNA to Akt-1 blocked c-FLIP protein expression, suggesting that Akt is an upstream mediator of c-FLIP. Furthermore, siRNA inhibition of either Akt-1 or c-FLIP enhanced $\alpha$-TEA-induced endoplasmic reticulum stress and endoplasmic reticulum stress-mediated upregulation of JNK/CHOP/DR5, indicating that suppression of prosurvival mediators by TAM $+\alpha$-TEA may enhance $\alpha$ TEA-induced endoplasmic reticulum stress-mediated JNK/CHOP/DR5, at least in part, via downregulation of activated Akt, which subsequently downregulates c-FLIP.

Based on published data and data present here, a schematic diagram of the known actions of $\alpha$-TEA, $\mathrm{M} \beta \mathrm{CD}$, and TAM on proapoptotic and prosurvival signaling in TAMR cells is depicted in Figure 7.

\section{Conclusions}

In summary, $\alpha$-TEA functions as a disruptor of cholesterol-rich lipid microdomains and an endoplasmic reticulum stress inducer in circumvention of TAMR. Although $\alpha$-TEA can effectively induce TAMR cells to undergo apoptosis as a single agent, it acts cooperatively with TAM at lower dosages to activate endoplasmic reticulum stress-mediated proapoptotic events and suppresses the highly amplified prosurvival signaling inherent in TAMR cells. As a potent anticancer agent, $\alpha$ TEA possesses several compelling features: (a) low toxicity to normal cells and tissues [12]; (b) dual anticancer actions (that is, suppresses prosurvival mediators and activates proapoptotic mediators); and (c) is effective against a wide range of cancer cell types with disparate molecular signatures [42]. Unlike single agents that target HER-1, HER-2, Akt, or mTOR, $\alpha$-TEA can inhibit multiple prosurvival mediators via disruption of cholesterol-rich lipid microdomains and induce apoptosis. These unique features of $\alpha$-TEA suggest great potential for use as a stand-alone adjuvant therapy or in combination with the adjuvant TAM for prevention and circumvention of TAMR in ER+ human breast cancers. Additionally, these data provide new knowledge about mechanisms of endocrine therapy resistance that may be useful in designing other agents for circumvention of TAMR.

\section{Abbreviations}

a-TEA: RRR-a-tocopherol ether-linked acetic acid analogue; Bcl-2: B-cell lymphoma 2; C-FLIP: cellular FLICE-inhibitory protein; CHOP: Ccaat-enhancer- binding protein (C/EBP) homologous protein; Cl: combination index; c-PARP: cleaved poly (ADP-ribose) polymerase; DAPI: 4'-6-diamidino-2-phenylindole; DilC-16: dialkylindocarbocyanine; DR5: death receptor 5; DR5 (L/S): death receptor 5 long/short; $\mathrm{EC}_{50}$ : half-maximal effective concentration; ED: effective dosages; ER: estrogen receptor; ER-a estrogen receptor-a ER+: estrogen receptor positive; FITC: fluorescein isothiocyanate; GAPDH: glyceraldehyde-3-phosphate dehydrogenase; GRP78: glucose-regulated protein-78; HER-1: epidermal growth factor receptor ErbB-1; HER-2: epidermal growth factor receptor-2 ErbB-2; IRS: insulin-receptor substrate; JNK: c-Jun Nterminal kinase; M $\beta C D$ : methyl- $\beta$-cyclodextrin; MCF-7/HER-2: clone 18 MCF-7 cells overexpressing HER-2; MCF-7/TAMR: acquired tamoxifen-resistant MCF7; MCF-7/TAMS: TAM-sensitive MCF-7/parental; mER: membrane estrogen receptor; mTOR: mammalian target of rapamycin; nER: nuclear estrogen receptor; pAkt: phosphorylated-Akt; PARP: poly(ADP-ribose) polymerase; PBS: phosphate-buffered saline; pERK1/2: phosphorylated-extracellular signalregulated kinases 1 and 2; pER-a (Ser-167): phosphorylated form of estrogen receptor-a at serine-167; pER-a (Ser-118): phosphorylated form of estrogen receptor-a at serine-118; pHER-1: phosphorylated-HER-1; pHER-2:

phosphorylated-HER-2; PI: propidium iodide; PI3K: phosphatidylinositol-3kinase; pJNK: phosphorylated-c-Jun N-terminal kinase; pmTOR:

phosphorylated mammalian target of rapamycin; RTKs: receptor tyrosine kinases; siRNA: small interfering RNA; TAM: tamoxifen; TAMR: tamoxifen resistant; TAMS: tamoxifen sensitive; tAkt: total Akt; tER-a total estrogen receptor-a tERK1/2: total extracellular signal-regulated kinases 1 and 2; tHER1: total HER-1; tHER-2: total HER-2; tBid: truncated Bid; VEH: vehicle control.

\section{Acknowledgements}

This work was supported by the Clayton Foundation for Research (BGS and KK), the Center for Molecular and Cellular Toxicology at the University of Texas, and the NIEHS/NIH Toxicology Training Grant T32 ES07247 (RT). We thank Dr. Shawn Bratton and Indra Mahajan (University of Texas at Austin) for providing Annexin V-FITC stain. The funding agencies have no role in study design; in the collection, analysis, and interpretation of data; in the writing of the manuscript; or in the decision to submit the manuscript for publication.

\section{Author details}

${ }^{1}$ School of Biological Sciences/A5000, University of Texas at Austin, 1 University Station, Austin, TX 78712, USA. ²Department of Nutritional Sciences/A2703, University of Texas at Austin, 1 University Station, Austin, TX 78712, USA.

\section{Authors' contributions}

WY, BGS, and KK conceived and designed the study, analyzed the data, and drafted the manuscript. RT performed all the experiments, conceived and designed the study, analyzed the data, and drafted the manuscript. LAD provided original MCF-7/TAMR and TAMS cells. All authors read and approved the manuscript for publication.

\section{Competing interests}

K Kline, BG Sanders, and W Yu are co-inventors on a-TEA patents, which are assigned to the Research Development Foundation (a nonprofit foundation that supports medical related research). A possibility exists that financial gain may be realized if a-TEA is successfully developed for clinical use. The authors declare that they have no competing interests.

Received: 7 April 2011 Revised: 6 June 2011

Accepted: 24 November 2011 Published: 24 November 2011

\section{References}

1. Jemal A, Siegel R, Xu J, Ward E: Cancer statistics. CA Cancer J Clin 2010, 60:277-300.

2. Fabian CJ: The what and how of aromatase inhibitors: hormonal agents for treatment and prevention of breast cancer. Clin Practice 2007, 12:2051-2063.

3. Lewis JS, Jordan VC: Selective estrogen receptor modulators (SERMs): mechanisms of anticarcinogenesis and drug resistance. Mutat Res 2005, 591:247-263.

4. Musgrove EA, Sutherland RL: Biological determinants of endocrine resistance in breast cancer. Nat Rev Cancer 2009, 9:631-643. 
5. Hurvitz SA, Pietras RJ: Rational management of endocrine resistance in breast cancer: a comprehensive review of estrogen receptor biology, treatment options, and future directions. Cancer 2008, 113:2385-2397.

6. Clarke R, Liu MC, Bouker KB, Gu Z, Lee RY, Zhu Y, Skaar TC, Gomez B, O'Brien K, Wang Y, Hilakivi-Clarke LA: Antiestrogen resistance in breast cancer and the role of estrogen receptor signaling. Oncogene 2003, 22:7316-7339.

7. Arpino G, Wiechmann L, Osborne CK, Schiff R: Crosstalk between the estrogen receptor and the HER tyrosine kinase receptor family: molecular mechanism and clinical implications for endocrine therapy resistance. Endocr Rev 2008, 29:217-233.

8. Shou J, Massarweh S, Osborne CK, Wakeling AE, Ali S, Weiss H, Schiff R: Mechanisms of tamoxifen resistance: increased estrogen receptor-HER2/ neu cross-talk in ER/HER2-positive breast cancer. J Natl Cancer Inst 2004, 96:926-935.

9. Li YC, Park MJ, Ye SY, Kim CW, Kim YN: Elevated levels of cholesterol-rich lipid rafts in cancer cells are correlated with apoptosis sensitivity induced by cholesterol-depleting agents. Am J Pathol 2006, 168:1107-1118.

10. Patra SK: Dissecting lipid raft facilitated cell signaling pathways in cancer. Biochim Biophys Acta 2008, 1785:182-206.

11. Hoessli DC, llangumaran S, Soltermann A, Robinson PJ, Borisch B, Nasir-UdDin: Signaling through sphingolipid microdomains of the plasma membrane: the concept of signaling platform. Glycoconj J 2000, 17:191-197.

12. Latimer P, Menchaca M, Snyder RM, Yu W, Gilbert BE, Sanders BG, Kline K: Aerosol delivery of liposomal formulated paclitaxel and vitamin $\mathrm{E}$ analog reduces murine mammary tumor burden and metastases. Exp Biol Med 2009, 234:1244-1252.

13. Jia $L, Y u W$, Wang $P$, Sanders BG, Kline $K$ : In vivo and in vitro studies of anticancer actions of alpha-TEA for human prostate cancer cells. Prostate 2008, 68:849-860.

14. Shun MC, Yu W, Gapor A, Parsons R, Atkinson J, Sanders BG, Kline K: Proapoptotic mechanisms of action of a novel vitamin $E$ analog (alpha-TEA) and a naturally occurring form of vitamin $E$ (delta-tocotrienol) in MDAMB-435 human breast cancer cells. Nutr Cancer 2004, 48:95-105.

15. Anderson K, Lawson KA, Simmons-Menchaca M, Sun L, Sanders BG, Kline K: Alpha-TEA plus cisplatin reduces human cisplatin-resistant ovarian cancer cell tumor burden and metastasis. Exp Biol Med 2004, 229:1169-1176.

16. Lawson KA, Anderson K, Menchaca M, Atkinson J, Sun L, Knight V, Gilbert BE, Conti C, Sanders BG, Kline K: Novel vitamin E analogue decreases syngeneic mouse mammary tumor burden and reduces lung metastasis. Mol Cancer Ther 2003, 2:437-444

17. Hahn T, Fried K, Hurley LH, Akporiaye ET: Orally active atocopheryloxyacetic acid suppresses tumor growth and multiplicity of spontaneous murine breast cancer. Mol Cancer Ther 2009, 8:1570-1578.

18. Tiwary R, Yu W, Li J, Park SK, Sanders BG, Kline K: Role of endoplasmic reticulum stress in alpha-TEA mediated TRAIL/DR5 death receptor dependent apoptosis. PLoS One 2010, 5:e11865.

19. Shun MC, Yu W, Park SK, Sanders BG, Kline K: Downregulation of epidermal growth factor receptor expression contributes to alpha-TEA's proapoptotic effects in human ovarian cancer cell lines. J Oncol 2010, 2010:824571.

20. Tiwary R, Yu W, Sanders BG, Kline K: a-TEA cooperates with MEK or mTOR inhibitors to induce apoptosis via targeting IRS/PI3K pathways. $\mathrm{Br}$ Cancer 2011, 104:101-109.

21. Yu W, Tiwary R, Li J, Park SK, Jia L, Xiong A, Simmons-Menchaca M, Sanders BG, Kline K: a-TEA induces apoptosis of human breast cancer cells via activation of TRAIL/DR5 death receptor pathway. Mol Carcinog 2010, 49:964-973.

22. Jia L, Yu W, Wang P, Li J, Sanders BG, Kline K: Critical roles for JNK, c-Jun, and Fas/FasL-signaling in vitamin $\mathrm{E}$ analog-induced apoptosis in human prostate cancer cells. Prostate 2008, 68:427-441.

23. Wang $P, Y u$ W, Hu Z, Jia L, lyer VR, Sanders BG, Kline K: Involvement of JNK/p73/NOXA in vitamin E analog-induced apoptosis of human breast cancer cells. Mol Carcinog 2008, 47:436-445.

24. Kline K, Lawson KA, Yu W, Sanders BG: Vitamin E and cancer. Vitam Horm 2007, 76:435-461.
25. Hahn T, Jagadish B, Mash EA, Garrison K, Akporiaye ET: aTocopheryloxyacetic acid: a novel chemotherapeutic that stimulates the antitumor immune response. Breast Cancer Res 2011, 13:R4.

26. Jayo A, Conde I, Lastres P, Martínez C, Rivera J, Vicente V, GonzálezManchón C: L718P mutation in the membrane-proximal cytoplasmic tail of beta 3 promotes abnormal alpha Ilb beta 3 clustering and lipid microdomain coalescence, and associates with a thrombasthenia-like phenotype. Haematologica 2010, 95:1158-1166.

27. Beknke O, Tranum-Jensen J, van Deurs B: Filipin as a cholesterol probe. I. Morphology of filipin-cholesterol interaction in lipid model systems. Eur J Cell Biol 1984, 35:189-199.

28. Chou T-C: Drug combination studies and their synergy quantification using the Chou-Talalay method. Cancer Res 2010, 70:440-446.

29. Pike LJ: Lipid rafts: bringing order to chaos. J Lipid Res 2003, 44:655-667.

30. Massarweh S, Schiff R: Resistance to endocrine therapy in breast cancer: exploiting estrogen receptor/growth factor signaling crosstalk. Endocr Relat Cancer Suppl 2006, 1:S15-S24.

31. Ghayad SE, Vendrell JA, Larbi SB, Dumontet C, Bieche I, Cohen PA: Endocrine resistance associated with activated ErbB system in breast cancer cells is reversed by inhibiting MAPK or PI3K/Akt signaling pathways. Int J Cancer 2010, 126:545-562.

32. deGraffenried LA, Friedrichs WE, Russell DH, Donzis EJ, Middleton AK, Silva JM, Roth RA, Hidalgo M: Inhibition of mTOR activity restores tamoxifen response in breast cancer cells with aberrant Akt Activity. Clin Cancer Res 2004, 10:8059-8067.

33. Leary AF, Drury S, Detre S, Pancholi S, Lykkesfeldt AE, Martin LA, Dowsett M, Johnston SR: Lapatinib restores hormone sensitivity with differential effects on estrogen receptor signaling in cell models of human epidermal growth factor receptor 2-negative breast cancer with acquired endocrine resistance. Clin Cancer Res 2010, 16:1486-1497.

34. Chu I, Blackwell K, Chen S, Slingerland J: The dual ErbB1/ErbB2 inhibitor, lapatinib (GW572016), cooperates with tamoxifen to inhibit both cell proliferation- and estrogen-dependent gene expression in antiestrogenresistant breast cancer. Cancer Res 2005, 65:18-25.

35. Nahta R, Esteva FJ: Herceptin: mechanisms of action and resistance. Cancer Lett 2006, 232:123-138.

36. Ferrer-Soler L, Vazquez-Martin A, Brunet J, Menendez JA, De Llorens R, Colomer R: An update of the mechanisms of resistance to EGFR-tyrosine kinase inhibitors in breast cancer: gefitinib (Iressa)-induced changes in the expression and nucleo-cytoplasmic trafficking of HER-ligands. Int J Mol Med 2007, 20:3-10.

37. Pike L: Growth factor receptors, lipid rafts and caveolae: an evolving story. Biochim Biophys Acta 2005, 1746:260-273.

38. Márquez DC, Chen HW, Curran EM, Welshons WV, Pietras RJ: Estrogen receptors in membrane lipid rafts and signal transduction in breast cancer. Mol Cell Endocrinol 2006, 246:91-100.

39. Freeman MR, Cinar B, Lu ML: Membrane rafts as potential sites of nongenomic hormonal signaling in prostate cancer. Trends Endocrinol Metab 2005, 16:273-279.

40. Li J, Yu W, Tiwary R, Park SK, Xiong A, Sanders BG, Kline K: a-TEA-induced death receptor dependent apoptosis involves activation of acid sphingomyelinase and elevated ceramide-enriched cell surface membranes. Cancer Cell Int 2010, 10:40

41. Yu C, Alterman M, Dobrowsky RT: Ceramide displaces cholesterol from lipid rafts and decreases the association of the cholesterol binding protein caveolin-1. J Lipid Res 2005, 46:1678-1691.

42. Anderson K, Simmons-Menchaca M, Lawson KA, Atkinson J, Sanders BG, Kline K: Differential response of human ovarian cancer cells to induction of apoptosis by vitamin E succinate and vitamin E analogue, alpha-TEA. Cancer Res 2004, 64:4263-4269.

doi: $10.1186 / \mathrm{bcr} 3063$

Cite this article as: Tiwary et al:: Targeting cholesterol-rich

microdomains to circumvent tamoxifen-resistant breast cancer. Breast Cancer Research 2011 13:R120. 